\title{
ENHANCEMENT OF NSLS SEXTUPOLES USING PERMANENT MAGNETS *
}

\author{
G. Rakowsky ${ }^{\dagger}$, BNL, Upton, NY 11973, USA
}

\begin{abstract}
The recent upgrade of the NSLS X-Ray Ring energy from 2.584 to $2.8 \mathrm{GeV}$ requires defocusing sextupole fields beyond the operating limits of the present sextupole magnets. We have demonstrated that the required fields can be achieved by addition of permanent magnets to counteract the saturation in the magnet poles. Magnetic measurements on a NSLS sextupole agree with fields predicted by Radia.
\end{abstract}

\section{INTRODUCTION}

With the upgrade of the NSLS X-Ray Ring energy from 2.584 to $2.8 \mathrm{GeV}$, the defocusing family of sextupole magnets must run at their thermal limit, at 800 amps. In this range the iron is heavily saturated. Still, the sextupole strength is insufficient to allow operation with positive chromaticity, which is desired for stable operation with higher beam currents. Increasing the current is not possible, since the magnets already run with aggressive cooling. Other ways to increase the sextupole strength by $10-20 \%$ were sought, short of replacing the magnets entirely.

Klaus Halbach first proposed the use of permanent magnets to counteract saturation in electromagnet-driven wigglers [1]. Rare-earth permanent magnet (PM) blocks placed between adjacent poles of the wiggler can shift the operating point of the iron downward along the B-H curve, improve the linearity and force some of the poleto-neighboring-pole leakage flux across the mid-plane, increasing the effective field of the wiggler. Halbach later extended the technique to other types of electromagnets, including multipoles [2].We report here our study of a similar use of permanent magnets to increase the strength of the NSLS sextupoles.

\section{3D MODELING}

The NSLS sextupole was modeled using the 3D magnetostatics code Radia [3]. This code uses a boundary integral method and makes use of analytical expressions to calculate fields and field integrals from currentcarrying coils, iron pieces and permanent magnets. Unlike finite-element codes, Radia does not require fine meshing of all of space, only a fairly coarse (but judicious) subdivision of the iron, to calculate fields of interest. The quadrupole example provided in the Radia distribution was used as a basis for the model. By applying symmetry,

\footnotetext{
*Work supported by the US Department of Energy under Contract DE-AC02-98CH10886

†Email: rakowsky@bnl.gov
}

only $1 / 24-$ th of the iron yoke and the permanent magnets, and only one of the six coils need to be modeled explicitly. The full model, as rendered by Radia, is shown in Figure 1.

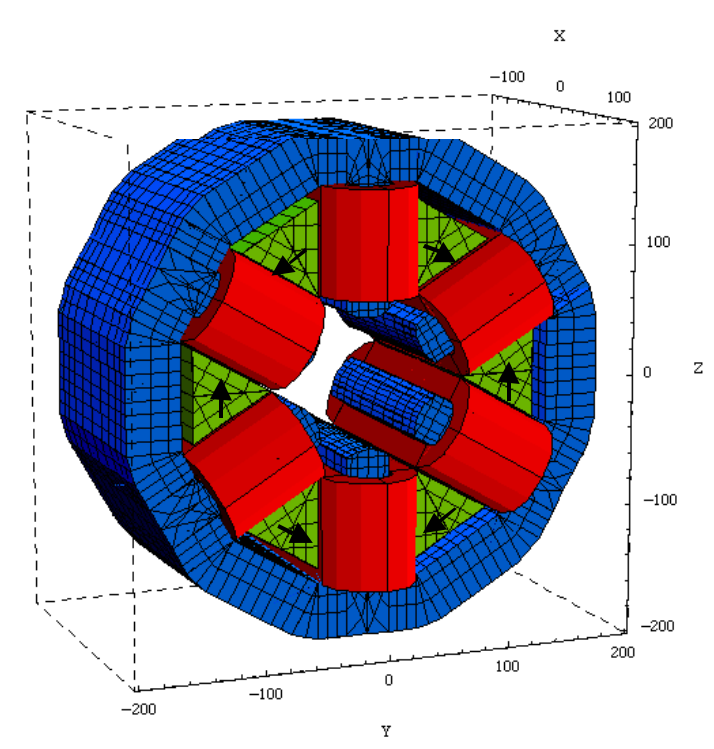

Figure 1. Radia rendering of the full NSLS Sextupole model with PM inserts. Arrows indicate relative directions of magnetization in the PM's.

\subsection{Field Calculations}

The Radia calculations confirm what has long been known about the NSLS sextupoles: that the heaviest saturation occurs in the stem and root of the pole, especially near the inside corner between the pole and the backleg. The poletip is far from saturation, so field quality is unaffected by saturation in the stem and root. The backleg is not heavily saturated either, since it has a considerably larger cross-section than the pole. A vector plot of the magnetizations in a slice through one pole sectant of the yoke at 800 amps is shown in Figure 2(a). The length of the arrows is proportional to the magnitude of the magnetization, with a maximum of about $2.1 \mathrm{~T}$ in the saturated region.

To counteract the saturation in the pole, PM inserts can be placed between adjacent poles, magnetized parallel to the backleg and oriented so as to drive flux through the pole and backleg opposite to the flux generated by the coils. Most of the PM flux returns through the lowreluctance path of the iron, with only a small fraction appearing in the airgap between the pole tips. 

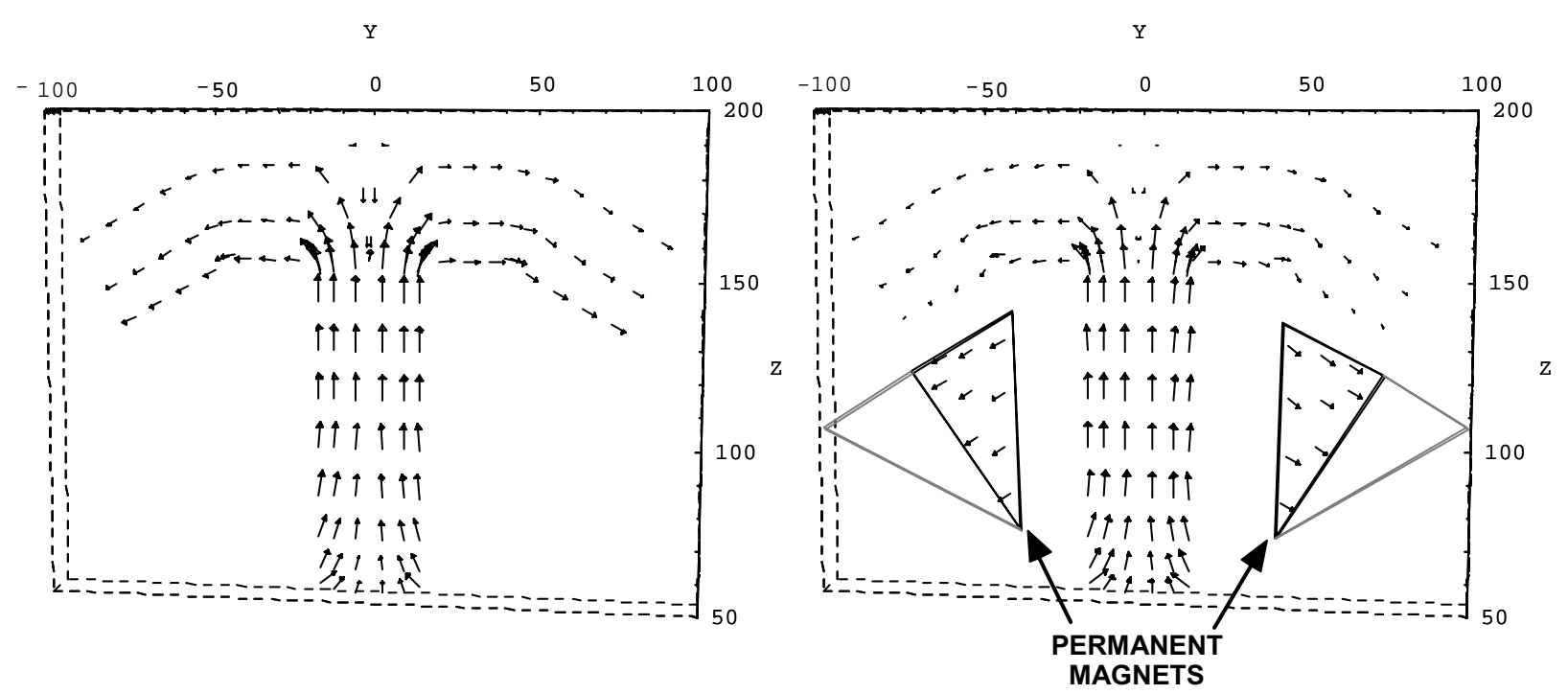

Figure 2. Vector magnetization plot in a slice through one sectant of an NSLS Sextupole at $800 \mathrm{~A}$, (a) without and (b) with PM inserts. Maximum magnetization in the pole is $2.1 \mathrm{~T}$. PM magnetization is $1.25 \mathrm{~T}$.

In the NSLS sextupole there are only two regions where a PM insert can be introduced: (a) in the triangular volume between adjacent coils and the backleg, and (b) in the pentagon-shaped space between the pole tips and the bottoms of the coils. Magnetically, Option (b) would have been preferred, because a much smaller volume of PM material would have been required, compared to Option (a). Unfortunately, the vacuum chamber intrudes into the space between the pole tips on one side, preempting that option. We therefore placed triangular PM inserts in the spaces between coils and the backlegs, seen in the rendering in Figure 1.

In the vector plot in Figure 2(b) one sees that with the $\mathrm{PM}$, magnetization in the iron is reduced, especially in the backleg. (At lower currents one finds that magnetization in the backleg is actually reversed.)

Radia calculations show that at zero current a small sextupole field appears in the gap, but it is well below the field required at the $750 \mathrm{MeV}$ injection energy. In the normal sextupole magnet the field vs. current response begins to exhibit saturation above 300 amps; with PM inserts the linear range is extended to about $450 \mathrm{amps}$. For currents $>300$ amps the fields in the gap are progressively higher with the PM inserts than without. At 800 amps the field is $20 \%$ higher with PM than without. (If both Options (a) and (b) had been available, the increase could have been nearly $40 \%$.) The results are summarized in Figure 3.

\section{MAGNETIC DESIGN}

A PM material with high remanent magnetization $\left(\mathrm{B}_{\mathrm{r}}\right)$ is desired. In the model described above, we used $\mathrm{NdFeB}$ with $\mathrm{B}_{\mathrm{r}}=1.25 \mathrm{~T}$. To estimate the intrinsic coercivity $\left(\mathrm{H}_{\mathrm{ci}}\right)$ required, we computed the field at 800 amps in the midplane, including the PM and the backleg. The field inside the $\mathrm{PM}$ itself ranges from -1 to $+1 \mathrm{~T}$, indicating the need for a material with a linear $\mathrm{B}-\mathrm{H}$ relationship throughout the $2^{\text {nd }}$ quadrant of the $\mathrm{B}-\mathrm{H}$ plane. This linearity must be maintained up to the maximum temperature to which the PM might be exposed in operation, about $65^{\circ} \mathrm{C}$. Therefore, a high-temperature grade of NdFeB with the "knee" of the B-H curve still in the $3^{\text {rd }}$ quadrant at $65^{\circ} \mathrm{C}$ is required. That translates into a value of $\mathrm{H}_{\mathrm{ci}}>17 \mathrm{kOe}$ at $20^{\circ} \mathrm{C}$. (For the prototype test we selected Shin-Etsu grade N38H, which meets these $B_{r}$ and $\mathrm{H}_{\mathrm{ci}}$ requirements, and is linear in the $2^{\text {nd }}$ quadrant up to $80^{\circ} \mathrm{C}$.)

\subsection{Magnetic Forces}

As a practical matter, we want to be able to retrofit the sextupoles with PM inserts in situ, if possible. That means the magnetic forces exerted on the PM blocks must be small enough to allow insertion or extraction of the PM by hand, or at most, with simple fixturing. For this reason, the PM must be maintained at some distance from the iron yoke at all times. The presence of the coils already assures separation of the PM from the poles on two sides. Separation of the third side from the backleg was accomplished by means of a 3/8-inch thick aluminum plate. Since the triangular PM inserts must be assembled from several smaller sub-blocks, the PM fabricator suggested gluing the PM to the aluminum plate as well. Triangular end covers retain the magnets in case of glue failure. A handle was attached to the baseplate to ease insertion and extraction. The baseplate represents a small loss of PM volume, but allows installation of the PM blocks safely by hand. The model calculations were made with allowance for this separation. 


\subsection{Field Errors}

Although the field profile in the aperture is dominated by the iron geometry, variations among PM blocks can introduce unwanted dipole and other multipole components. In our case the PM contributes an increase of about $20 \%$ in sextupole strength at 800 amps, so errors in PM magnetization will have a second-order effect on the total field. For example, for a $3 \%$ increase in $\mathrm{B}_{\mathrm{r}}$, Radia calculates a $0.4 \%$ increase in sextupole field. Limiting the variation in $\mathrm{B}_{\mathrm{r}}$ to $\pm 3 \%$ will limit non-sextupole fields to less than $\pm 1 \%$. A similar argument can be made about errors in magnetization angle.

In recent years, PM manufacturers have improved the control of magnetic properties. Magnetization tolerances of $\pm 1 \%$ and $\pm 1^{\circ}$ are now routinely met. In fact, the six prototype PM inserts were found to vary by less than $\pm 0.25 \%$ and $\pm 0.8^{\circ}$. Variation among the PM inserts within each sextupole can be further reduced by sorting.

Temperature dependence of $\mathrm{B}_{\mathrm{r}}$ needs to be considered as well since the PM inserts are in close proximity to the coils whose temperature varies during injection and ramping. The PM manufacturer's data shows that between $20^{\circ}$ and $80^{\circ} \mathrm{C}, \mathrm{B}_{\mathrm{r}}$ decreases by $4.8 \%$. This leads to a reduction in sextupole field of less than $0.7 \%$, an acceptably small error.

\section{FIELD MEASUREMENTS}

A spare NSLS sextupole magnet was fitted with a Hall probe in the gap between adjacent poles. Field readings were taken over the range $0-800 \mathrm{amps}$, both with and without the PM inserts. The measurement data are plotted in Figure 3. The solid and dashed curves are Radia calculations. The agreement between simulation and measurement is quite good. Differences can be attributed to (1) slightly higher $B_{r}$ than expected, (2) differences between the actual characteristic of the 1001 steel used in the NSLS sextupole and the built-in B-H data for grade 1006 steel available in Radia, and (3) the somewhat coarse segmentation of the iron in the Radia model. Still planned are rotating coil measurements of the integrated multipoles to assess the contribution of various PM errors to unwanted multipole components.

\section{CONCLUSIONS}

The use of permanent magnets to counteract saturation effects and to extend the operating range of conventional multipoles has been demonstrated. Field measurements in an NSLS sextupole magnet agree well with fields calculated by the 3D code Radia and confirm the predicted $20 \%$ increase in sextupole field. The PM inserts can be handled by hand and allow a fairly simple retrofit.

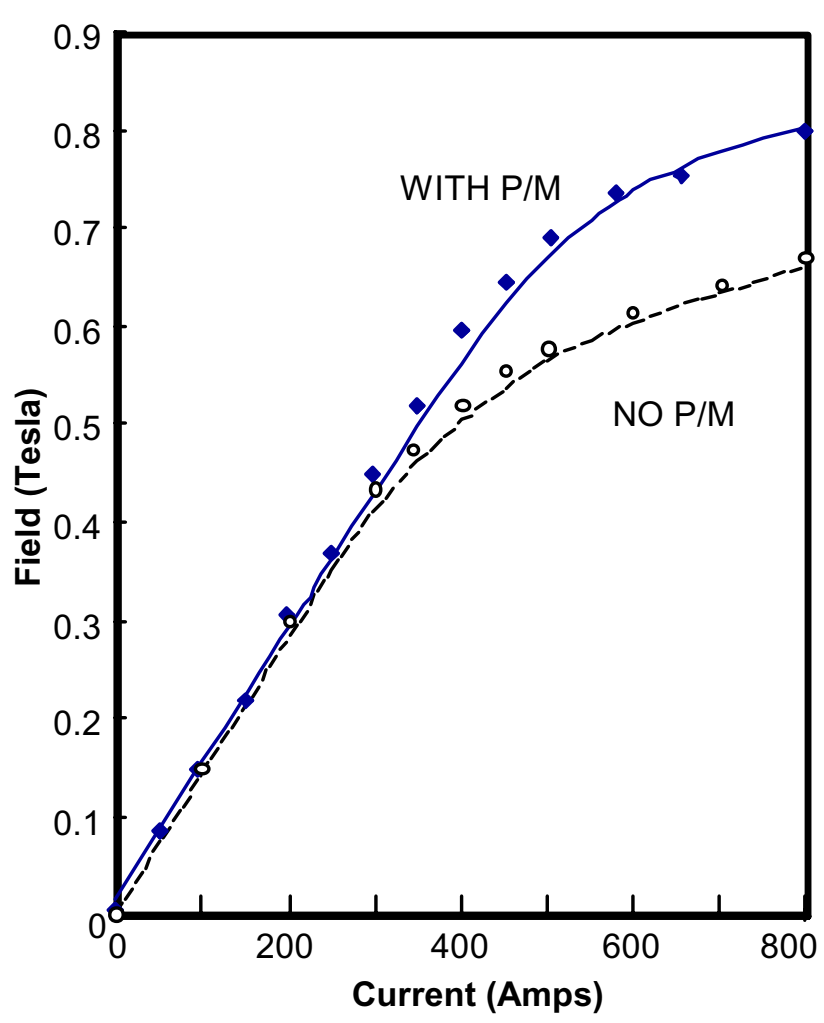

Figure 3. Peak field between adjacent poles vs. current, without and with PM. The curves are Radia simulations.

\section{ACKNOWLEDGEMENT}

The idea of extending the performance of the NSLS sextupoles with permanent magnets was first proposed independently by Eric Blum. His helpful suggestions and a critical reading of this manuscript are gratefully acknowledged. Special thanks to Michael Lehecka for technical assistance, and also to Pete Zuhoski, Mike Fulkerson and other NSLS staff for help in conducting the measurements. The PM inserts were fabricated by Magnet Sales \& Manufacturing, Culver City, CA.

\section{REFERENCES}

[1] K. Halbach, "Some Concepts to Improve The Performance of DC Electromagnetic Wigglers", Proc 7th FEL Conf., 1985, Granlibakken, CA. Also published as Lawrence Berkeley Laboratory Report LBL-20502.

[2] K. Halbach, "Specialty Magnets", Proc. AIP Summer School of Particle Accelerators, Palo Alto, CA, 1986. Also published as LBL Report LBL-21945.

[3] P. Elleaume, O. Chubar, J. Chevanne, "Computing 3D Magnetic Fields from Insertion Devices", PAC'97, Vancouver, BC, Canada; p. 3509-3511. 